\title{
Recomendaciones para la reapertura de servicios de cirugía electiva durante la pandemia por SARS-CoV-2
}

\author{
Fredy Orlando Mendivelso Duarte ${ }^{1}$, Milena Rodríguez Bedoya ${ }^{2}$ y Arnold José Barrios Parra ${ }^{1}$
}

Forma de citar

Mendivelso Duarte FO, Rodríguez Bedoya M, Barrios Parra AJ. Recomendaciones para la reapertura de servicios de cirugía electiva durante la pandemia por SARS-CoV-2 Rev Panam Salud Publica. 2020;44:e114. https://doi.org/10.26633/ RPSP.2020.114

RESUMEN

Dada la incertidumbre que acompaña a la pandemia por SARS-CoV-2 y ante la necesidad de dar respuesta a múltiples problemas de salud crónicos y agudos que afectan a la población general, incluidos aquellos que precisan de una intervención quirúrgica, se presentan las recomendaciones implementadas en clínicas y hospitales de Colombia como guía para lograr una reapertura de los servicios de cirugía electiva de forma segura, escalonada y monitoreada acorde a la dinámica que impone la pandemia, la regulación nacional e internacional y la velocidad en la producción de evidencia científica relacionada con la COVID-19.

Palabras clave Infecciones por coronavirus; procedimientos quirúrgicos ambulatorios; virus del SRAS; quirófanos; cirugía general; Colombia; América Latina.

Los pacientes en quienes la cirugía se considera "electiva" son aquellos con problemas agudos y crónicos cuya intervención quirúrgica ciertamente puede retrasarse sin un daño significativo para el paciente o el resultado final. Aunque se puede argumentar la necesidad de cirugía en algunas personas debido al dolor o al deterioro funcional, el principio determinante es que retrasar el tratamiento no alterará significativamente el resultado final $(1,2)$. La pandemia ocasionada por el SARSCoV-2 y la enfermedad resultante (coronavirus 2019 disease, COVID-19), llevó en todo el mundo a cambios significativos en la forma de prestar los servicios de salud, incluida la actividad quirúrgica. La situación epidemiológica de la pandemia en cada país es diferente, y cada semana se modifica la incidencia de casos, muertes y pacientes recuperados (3). En algunos países se está alcanzando el denominado pico epidemiológico de la pandemia, mientras que en otros ya se vislumbra un descenso en el número de casos. Dado lo anterior y las recientes directrices gubernamentales orientadas al reinicio por fases de las actividades comerciales y económicas denominadas esenciales
(4), desde la prestación de servicios de salud se debe buscar un equilibrio entre las necesidades económicas y de salud pública frente al reto de reiniciar los procedimientos que se consideran electivos de una manera que no se afecten los sistemas de salud en tiempos de pandemia pero que tampoco se afecte la salud y la calidad de vida de las personas que tienen indicación de un procedimiento quirúrgico (5).

Las diferentes proyecciones muestran que el SARS-CoV2 continuará afectando a las poblaciones por un periodo largo aún no establecido. Según modelos matemáticos, los brotes recurrentes pueden llegar hasta el año 2024 o 2025; se menciona incluso que las medidas de aislamiento físico se deberían prolongar por lo menos hasta 2022 (6). Muchos procedimientos de cirugía electiva fueron suspendidos al inicio de la pandemia acatando las recomendaciones de protección individual y el distanciamiento físico para proteger a niños, adultos mayores y personas con comorbilidades definidas como de riesgo para la COVID-19. También se acataron en gran medida para proteger y preparar la respuesta de los servicios de salud. Como consecuencia

\footnotetext{
1 Clínica Reina Sofía, Bogotá, Colombia. $₫$ Fredy Mendivelso, mendivelsofredy@ hotmail.com
}

\footnotetext{
2 Instituto Nacional de Salud, Bogotá, Colombia
} 
directa de estas medidas acertadas y tomadas en el momento indicado por las autoridades nacionales, es probable que la demanda acumulada de procedimientos quirúrgicos pueda ser inmensa y las instituciones, los médicos y otros integrantes de los equipos de salud deben estar preparados para satisfacer dicha demanda durante la pandemia. Tomar la decisión de programar nuevamente una cirugía electiva obedece a múltiples propósitos; sin embargo, hay varios lineamientos que deben ser tenidos en cuenta para lograr un proceso exitoso mientras continúe la pandemia (6).

En este artículo se sintetizan las recomendaciones basadas en la mejor evidencia disponible hasta el momento y las lecciones aprendidas durante el proceso de reapertura de servicios de cirugía electiva de alta complejidad, cirugía ambulatoria mayor y cirugía de corta estancia instaurados en instituciones de mediana y alta complejidad en Colombia tomando como marco de referencia el riesgo individual de infección por COVID-19, la disminución de casos en el área geográfica de influencia de cada hospital y el riesgo de que pueda empeorar la salud de los pacientes que requieren una cirugía electiva si se demora la intervención.

\section{ALISTAMIENTO INSTITUCIONAL}

- Tanto la apertura como la decisión de suspender la programación de procedimientos de cirugía electiva se regirán por decisiones informadas con datos derivados del monitoreo diario institucional, contrastados y acatando las alertas dadas por las autoridades locales y nacionales. Para que dicho monitoreo sea efectivo y eficiente, cada institución conformará un centro de mando que puede ser denominado Comité Multidisciplinario de Quirófanos (CMQ); este es un comité asesor de las decisiones que con responsabilidad ejecutiva recaen tanto en el director científico como en la gerencia de cada clínica u hospital durante la pandemia. Se recomienda que el CMQ incluya líderes de áreas quirúrgicas, anestesia y cuidado crítico, infectología, epidemiología, salud mental, bioética y asociación de pacientes.

- Las funciones de los CMQ son la recolección, el monitoreo, el análisis y la evaluación diaria del avance y comportamiento del plan de reactivación de cirugía electiva, incluida la decisión de programar y cancelar procedimientos. También se encargará de dar a conocer y promover el cumplimiento de las normas e informar las dudas o situaciones que se presenten durante el proceso de reactivación facilitando su gestión y resolución.

- Es prioritario que el CMQ incluya las cifras de disponibilidad de camas en la unidad de cuidados intensivos (UCI) dado que con el incremento proyectado en el número de casos es el indicador más fiable de la capacidad de respuesta institucional y de la red hospitalaria. Aunque la oferta de camas UCI se ha incrementado por los planes de expansión en clínicas y hospitales, también es necesario contrastarla con el indicador de personal idóneo para su operación.

- Considerar siempre la ocurrencia de casos de emergencia quirúrgica y prever la capacidad de las instalaciones para su atención.

- La programación de una cirugía estará condicionada a la evaluación y el seguimiento del recurso hospitalario, al comportamiento de la pandemia y a las decisiones de tipo administrativo y de salud pública que se generen a partir de las autoridades competentes (Figura 1). Debe determinarse el número de profesionales de los equipos quirúrgicos y de apoyo sospechosos o confirmados para la COVID-19 y el número de pacientes llevados a cirugía electiva que durante el posquirúrgico inmediato de 14 días son reportados como positivos para la COVID-19. En ambos casos, se requiere realizar de inmediato la investigación de campo para identificar los contactos efectivos en la institución y apoyarse con las autoridades de salud y de aseguramiento en la búsqueda activa, el tamizaje, la atención y la protección de contactos ambulatorios.

- Cada institución debe censar y conocer las comorbilidades y la edad de los miembros del equipo quirúrgico con el fin de estratificar sus riesgos y establecer las medidas de protección apropiadas en forma individual.

- Instaurar una rotación del personal de quirófanos y áreas de apoyo para evitar la sobreexposición.

- Establecer procesos de seguimiento y apoyo para el personal de salud definiendo estrategias de detección de conductas emocionales atípicas que permitan hallar alteraciones del estado emocional, fatiga y riesgo de burnout.

- Flexibilizar los horarios del personal con afectación emocional o con familiares contagiados (4,7-9).

\section{FASE PREQUIRÚRGICA}

- Evaluar las listas de pacientes cancelados y priorizar su atención según su patología quirúrgica de base (se recomienda el uso de la escala de estratificación MeNTS-Medically Necessary Time Sensitive)(10).

- Organizar bloques quirúrgicos que puedan realizarse con anestesia local o regional para reducir la generación de aerosoles.

- Los procedimientos diagnósticos ordenados deben realizarse según se consideren domiciliarios o ambulatorios cumpliendo medidas de bioseguridad.

- En todo paciente con indicación de cirugía electiva, documentar de manera estricta los antecedentes de comorbilidades como inmunosupresión, VIH, cáncer, quimioterapia, diabetes, hipertensión, obesidad, falla renal, hipotiroidismo, malnutrición, uso de corticoides, enfermedad pulmonar obstructiva crónica/asma y tabaquismo; estos pacientes tienen mayor riesgo de ingreso a la UCI y de morbimortalidad en caso de resultar afectados por la COVID-19.

- Diseñar y diligenciar a todo paciente programado para cirugía electiva un consentimiento informado sobre el riesgo de COVID-19.

- No se recomienda la formulación de "dispensa laboral" para dar cumplimiento a medidas de autocuidado previo a la cirugía.

- El paciente y su familiar deben conocer con la debida anticipación la ruta de acceso y las estaciones de control necesarias para lograr un rápido ingreso al quirófano y los controles administrativos.

- Inhabilitar las salas de espera; de esta manera la visita al paciente se concentrará en su habitación y no en áreas comunes (solamente debe permitirse un acompañante).

- Velar por el adecuado estado de los equipos que minimicen la exposición al gas o el humo (neumoinsufladores con filtros) o pantallas que mejoren la visión en presencia de humo para los equipos quirúrgicos. 
FIGURA 1. Indicadores de referencia para el manejo de intervenciones quirúrgicas programadas en instituciones de salud

\begin{tabular}{|c|c|c|c|c|}
\hline \multirow{3}{*}{ Escenarios } & \multicolumn{4}{|c|}{ Fase } \\
\hline & I & II & III & IV \\
\hline & Alerta mínima & Alerta leve & Alerta media & Alerta alta \\
\hline $\begin{array}{l}\text { Porcentaje de } \\
\text { ocupación en UCI } \\
\text { con pacientes } \\
\text { CoVID-19[+] }\end{array}$ & $<15 \%$ & $15-29 \%$ & $30-70 \%$ & $>70 \%$ \\
\hline $\begin{array}{l}\text { Porcentaje de } \\
\text { ocupación en } \\
\text { hospitalización con } \\
\text { pacientes } \\
\text { COVID-19[+] }\end{array}$ & $<10 \%$ & $10-29 \%$ & $30-60 \%$ & $>60 \%$ \\
\hline $\begin{array}{l}\text { Decisión sobre la } \\
\text { actividad quirúrgica }\end{array}$ & Normal & $\begin{array}{c}\text { Pacientes que } \\
\text { podrían requerir } \\
\text { UCI }\end{array}$ & $\begin{array}{l}\text { Cirugía de mínimo } \\
\text { riesgo de UCI, } \\
\text { teniendo pisos } \\
\text { exclusivos No } \\
\text { COVID-19 } \\
\text { disponibles }\end{array}$ & $\begin{array}{l}\text { Solo cirugía } \\
\text { prioritaria y } \\
\text { cirugía de } \\
\text { urgencia no } \\
\text { diferible }\end{array}$ \\
\hline
\end{tabular}

- Restringir el uso del vestido de calle y de calzado dentro de la institución hospitalaria, donde debe ser utilizado el vestido y calzado de dotación, y evitar el uso de gorros extrainstitucionales.

- Minimizar el uso de dispositivos que generen humo y aerosoles.

- Asegurar en instituciones con cirugía ambulatoria mayor un plan de atención quirúrgica para la reanudación de las actividades, que garantice un adecuado proceso de evaluación y de selección de pacientes que incluya, como mínimo, cuestionarios para la evaluación de signos y síntomas relacionados a la infección por COVID-19.

- Poner en marcha mecanismos o recordatorios 72 o 48 horas antes para disminuir el abandono de la cita planeada para la cirugía $(2,7,9,10)$.

\section{TAMIZAJE DE COVID-19}

- Buscar la presencia de fiebre, odinofagia, anosmia, disgeusia, tos seca, disnea, adinamia o fatiga, síntomas gastrointestinales (náusea, diarrea), antecedente de confinamiento, o contacto en los últimos 14 días con paciente sospechoso/ confirmado COVID-19. La presencia de cualquiera de los síntomas o contactos mencionados constituyen un tamizaje clínico [+] y se debe cancelar la programación del procedimiento, recomendar aislamiento físico por 14 días, solicitar estudios de PCR-TR y, de acuerdo con el resultado de la pruebas y la resolución de los síntomas, evaluar al paciente una vez se encuentre completamente recuperado para programar su cirugía.

- Frente a un tamizaje clínico [-] y si la institución cuenta con pruebas rápidas IgG e IgM se recomienda realizarlas 24 horas antes de la cirugía o de acuerdo al protocolo particular de la institución, con la siguiente interpretación:

- Paciente IgM [-] con IgG [+] (recuperado), apto para cirugía.

- Paciente IgM [-] con IgG [-] (sano sin inmunidad), apto para cirugía.

- Paciente IgM [+] con IgG [+] o IgM [+] con IgG [-] (infección activa), se debe cancelar el procedimiento y se definirá la realización de una PCR-TR.

- Verificar que se realice tamizaje al personal del área quirúrgica de acuerdo con las pruebas y la periodicidad definida por cada institución (8-10).

\section{ESTRATIFICACIÓN DE RIESGO}

- Después de la aplicación de la escala MeNTS y en presencia de un puntaje $\geq 55$ puntos, reconsiderar la realización del procedimiento quirúrgico. Debe destacarse que la aplicación de la escala no reemplaza el juicio clínico del cirujano; más bien supone una evaluación detallada de cada paciente que permita la toma de decisiones conjunta con este, quien debe tener muy claros los riesgos particulares de ser operado durante la pandemia y así debe quedar registrado en la historia clínica.

- En casos particulares será útil citar una junta de varios especialistas para la discusión del caso, y en situaciones de mayor complejidad se debe acudir a la instancia de un comité de ética $(7,8,10)$.

\section{PRIORIZACIÓN Y PROGRAMACIÓN DE CASOS}

El CMQ definirá y modificará la distribución de las jornadas quirúrgicas con el objeto de establecer la programación de los 
casos que deben realizarse teniendo en cuenta la prioridad, la complejidad quirúrgica y el estado del paciente, considerando asimismo la fase de la pandemia o el escenario en que se encuentre la institución. El CMQ definirá los horarios teniendo en cuenta la recomendación de prolongar los tiempos de programación en salas, por lo cual se puede considerar la extensión del tiempo de funcionamiento durante las horas de la noche y los fines de semana para responder a la demanda de cirugía electiva. Se recomienda mantener una programación escalonada para evitar aglomeraciones (7).

\section{EQUIPOS DE PROTECCIÓN PERSONAL (EPP)}

- Toda institución deberá adherirse a las definiciones establecidas por las autoridades nacionales sobre la calidad, la disposición y el uso adecuado de los EPP. Entender los mecanismos de transmisión del virus es muy importante, tanto para el manejo de los pacientes como para el adecuado uso de los EPP.

- Evaluar a corto y mediano plazo la disponibilidad de EPP tomando en cuenta el aumento exponencial de casos y la posibilidad de una segunda ola de la pandemia, así como escenarios de desabastecimiento del mercado.

- La institución debe garantizar la disponibilidad y protocolizar el uso, retiro y destino final de todos los EPP, tanto para la protección contra gotas y aerosoles como los necesarios para disminuir el riesgo por contacto. Lo anterior es una medida complementaria al mantenimiento de conductas individuales y colectivas de autoprotección (cultura de seguridad y calidad) sumada a los protocolos de limpieza e higiene exhaustivos de equipos e infraestructura.

- Incentivar el reporte de signos y síntomas, así como de la ocurrencia de contactos estrechos no protegidos con pacientes confirmados o sospechosos para la COVID-19 $(8,9)$.

\section{FASE QUIRÚRGICA}

- Realizar la cirugía electiva de pacientes sin COVID-19 en circuitos completamente diferenciados desde el ingreso a las clínicas hasta el egreso, a través de habitaciones en pisos, camas en UCI, unidades de cuidado posanestesia o cuidados intermedios diferenciadas físicamente y quirófanos preferenciales limpios y seguros. Los quirófanos de urgencias y para pacientes con COVID-19 deben estar claramente definidos.

- Determinar según el tipo de salas, el recambio del aire que permita definir el tiempo entre procedimientos y los momentos de circulación en el quirófano.

- Establecer protocolos de verificación de equipos y cambio de filtros de los sistemas de ventilación.

- Recomendar la disponibilidad de personal de apoyo para circular los diferentes quirófanos, desestimando la entrada y salida continua de la circulante de la sala, disminuyendo así la turbulencia del quirófano y la posibilidad de contaminación cruzada.

- Definir los protocolos quirúrgicos y anestésicos antes de iniciarse el procedimiento para disminuir la rotación de elementos en la sala.

- Definir con el servicio de patología las normas de embalaje y transporte de las muestras, y suprimir el uso de biopsias por congelación durante la pandemia.
- Vigilar y exigir el cumplimiento de las listas de seguridad para el paciente.

- Respecto del abordaje quirúrgico (cirugía abierta o laparoscópica), el cirujano elegirá la vía de acuerdo con su pericia. No existe evidencia que permita recomendar el no hacer cirugía laparoscópica. Debe monitorizarse la información con relación a los dispositivos de energía avanzada, que a la fecha no es concluyente.

- En la cirugía laparoscópica se recomienda el uso de un circuito cerrado de eliminación del gas y del humo para disminuir el riesgo de exposición.

- Emplear presiones bajas de neumoperitoneo, para lo cual es indispensable una adecuada relajación del paciente.

- Preferir trocares desechables, que brindan un sello más hermético.

- Realizar incisiones pequeñas ajustadas al trocar.

- Manipular cuidadosamente los instrumentos.

- En instituciones que cuenten con médicos residentes de especialidades quirúrgicas será responsabilidad del cirujano tratante la delegación de funciones, y decidir el momento y caso que puede asumir el residente en entrenamiento cumpliendo las mismas recomendaciones de cuidado de todo el equipo quirúrgico.

- Extraer trocares y especímenes quirúrgicos después de evacuar por completo el neumoperitoneo.

- Se recomienda el cierre de las heridas quirúrgicas con material que no requiera ser retirado (como cianoacrilato o suturas monofilamento absorbibles a corto plazo), así como evitar el uso de drenes o mechas, lo cual facilitará el control no presencial del paciente $(7,8)$.

\section{FASE POSQUIRÚRGICA}

- Se recomienda que el control posquirúrgico se realice de manera virtual cuando sea posible en los tiempos que determinen los protocolos institucionales.

- Cada institución generará instructivos para los pacientes, quienes deben mantener un aislamiento preventivo por dos semanas en el período posquirúrgico $(2,5,7)$.

\section{SEGUIMIENTO POSEGRESO}

- Cada institución deberá realizar un seguimiento de los pacientes de cirugía electiva hasta 14 días posteriores a la fecha de la cirugía, enfatizando la posibilidad de detectar pacientes que se contagiaron con SARS-CoV-2.

- Garantizar un adecuado seguimiento posoperatorio, incluido un programa de atención domiciliaria en los casos que se requiera y que se disponga del servicio, o coordinar dicho seguimiento con las autoridades locales de salud $(8,9)$.

\section{EVALUACIÓN}

- Durante la pandemia, salvo atenuantes mayores, el CMQ realizará sus actividades de forma diaria; debe adoptar medidas suficientes que garanticen la continuidad de su funcionamiento $(2,8)$.

- La revisión constante de literatura científica bajo los preceptos de la medicina basada en evidencia es una estartegia fundamental para informar la toma de decisiones durante la pandemia. 
Contribución de los autores. Todos los autores (FM, MR, AB) concibieron el estudio original, recolectaron consolidaron $\mathrm{y}$ analizaron los datos, interpretaron los resultados, escribieron y revisaron el manuscrito, atendieron la solicitud de ajustes realizadas por los evaluadores, revisaron y aprobaron la versión final.
Conflicto de intereses. Ninguno declarado.

Declaración. Las opiniones expresadas en este manuscrito son responsabilidad de los autores y no reflejan necesariamente los criterios ni la política de la RPSP/ PAJPH o de la OPS.

\section{REFERENCIAS}

1. American College of Surgeons. COVID-19 and Surgery. [Internet]. American College of Surgeons. 2020 [cited 2020 May 8]. Disponible en: https://www.facs.org/

2. Rajan N, Joshi GP. The COVID-19: Role of Ambulatory Surgery Facilities in This Global Pandemic. Anesth Analg. 2020 Apr (Online ahead of print). doi: 10.1213/ANE.0000000000004847.

3. Johns Hopkins University. Coronavirus COVID-19 (2019-nCoV). Dashboard by the Center for Systems Science and Engineering (CSSE) at Johns Hopkins University (JHU). [Internet]. 2020 [citado 2020 Jul 10]. Disponible en: https://gisanddata.maps.arcgis.com/apps/ opsdashboard/index.html\#/bda7594740fd40299423467b48e9ecf6

4. Ministerio de Salud y Protección Social de Colombia. Plan de acción para la prestación de servicios de salud durante las etapas de contención y mitigación de la pandemia por sars-cov-2 (covid-19) [Internet]. 2020 [citado 2020 Jun 20]. Disponible en: https://www.minsalud.gov.co/Ministerio/Institucional/Procesos y procedimientos/PSSS01.pdf

5. Ministerio de Salud y Protección Social de Colombia. Orientaciones para la restauración gradual de los servicios de salud en las fases de mitigación y control de la emergencia sanitaria por Covid-19 en Colombia. [Internet]. 2020 [citado 2020 Jun 20]. p. 1-17. Disponible en: https://www.minsalud.gov.co/Ministerio/Institucional/ Procesos y procedimientos/PSSS05.pdf

6. Kissler SM, Tedijanto C, Goldstein E, Grad YH, Lipsitch M. Projecting the transmission dynamics of SARS-CoV-2 through the postpandemic period. Science. 2020 Apr 14. doi: 10.1126/science. abb5793.

7. Barrios A, Prieto R, Torregrosa L, Álvarez C, Hernández J, Mendivelso $\mathrm{F}$, et al. Volver a empezar: cirugía electiva durante la pandemia del SARS-CoV2. Recomendaciones desde la Asociación Colombiana de Cirugía. Rev Colomb Cirugía. 2020;35(2):302-321. doi:10.30944/20117582.656.

8. American College of Surgeons. American Society of Anesthesiologists. Association of periOperative Registered Nurses. American Hospital Association. Joint Statement: Roadmap for Resuming Elective Surgery after COVID-19 Pandemic [Internet]. 2020 [citado 2020 May 1]. p. 1-4. Disponible en: https:// www.asahq.org/about-asa/newsroom/news-releases/2020/04/ joint-statement-on-elective-surgery-after-covid-19-pandemic

9. Asociación Colombiana de Infectología. ACIN. Instituto de Evaluación de Tecnologías en Salud. IETS. Consenso colombiano de atención, diagnóstico y manejo de la infección por SARS-COV-2/ COVID 19 en establecimientos de atención de la salud. Infectio. 2020;24(3):1-153. doi:10.22354/in.v24i3.851.

10. Prachand VN, Milner R, Angelos P, Posner MC, Fung JJ, Agrawal $\mathrm{N}$, et al. Medically Necessary, Time-Sensitive Procedures: Scoring System to Ethically and Efficiently Manage Resource Scarcity and Provider Risk During the COVID-19 Pandemic. J Am Coll Surg. 2020 Apr;1-8. doi: 10.1016/j.jamcollsurg.2020.04.011.

Manuscrito recibido el 24 de mayo de 2020. Aceptado para publicación, tras revisión, el 17 de julio de 2020

\section{Recommendations for reopening elective surgery services during the SARS-CoV-2 pandemic}

ABSTRACT Given the uncertainty that accompanies the SARS-CoV-2 pandemic and the need to respond to multiple chronic and acute health problems affecting the general population, including those requiring surgical intervention, the recommendations implemented in clinics and hospitals in Colombia are presented as a guide to achieve a reopening of elective surgery services in a safe, staggered and monitored manner in accordance with the dynamics imposed by the pandemic, national and international guidelines and the speed of production of scientific evidence related to COVID-19.

Keywords Coronavirus infections; ambulatory surgical procedures; SARS virus; operating rooms; general surgery; Colombia; Latin America. 